DOI: 10.12731/wsd-2018-5-108-118

УДК 616.127

\title{
ОСОБЕННОСТИ \\ ПОЛИМОРФИЗМА ГЕНОВ ИЛ-6 (IL-6-174G/C; \\ IL-6-572G/C) У ПАЦИЕНТОВ РАЗЛИЧНЫХ ЭТНИЧЕСКИХ ГРУПП С ИШЕМИЧЕСКОЙ БОЛЕЗНЬЮ СЕРДЦА (ОБЗОР ЛИТЕРАТУРЫ)
}

\section{Смирнова О.В., Каблуков В.С.}

Цель исследования. Представить читателю подробный обзор особенностей полиморфизма генов ИЛ-6 у пачиентов различных этнических групп с ишемической болезнью сердия (ИБС), а также исследовать влияние разных генотипов однонуклеотидных полиморфизмов интерлейкина ИЛ-6 на циркуляционные уровни воспалительных и противовоспалительных циитокинов у пациентов с различными частотами аллелей с ИБС.

Материал и методы исследования. Авторами был проведён поиск литературы об особенностях полиморфизма генов ИЛ-б у пациентов различных этнических групп с ИБС, с использованием соответствующих ключевых слов, в поисковых системах PubMеd и Google Scholar, по базам daнных Scopus, Web of Science, MedLine, The Cochrane Library, EMBASE, Global Health, CyberLeninka, РИНЦ и другим.

Результаты. Потенииальная связь между полиморфизмами генов ИЛ-6 и ИБС широко исследованы на сегодняшний день, однако результаты исследований остаются непоследовательными и несколько противоречивыми. Наиболее сильная взаимосвязь установлена по генотипам IL-6-174G/C; IL-6-572G/C для различных этнических групn, что и обуславливает интерес изучения в этом направлении в эпоху индивидуализации медицины.

Область применения результатов. Организация здравоохранения, кардиология, внутренние болезни, патологическая физиология.

Ключевые слова: ИЛ-6; полиморфизм генов; ичтоокиновая регуляция; ишемическая болезнь сердиа; атеросклероз. 


\title{
FEATURES OF GENE POLYMORPHISMS OF IL-6 (IL-6-174G/C IL-6-572G/C) IN PATIENTS OF DIFFERENT ETHNIC GROUPS WITH CORONARY HEART DISEASE (LITERATURE REVIEW)
}

\author{
Smirnova O.V., Kablukov V.S.
}

The aim of the study: to Provide the reader with a detailed overview of the features of IL-6 gene polymorphism in patients of different ethnic groups with coronary heart disease (CHD), as well as to investigate the effect of different genotypes of single-nucleotide polymorphisms of interleukin IL-6 on the circulation levels of inflammatory and anti-inflammatory cytokines in patients with different frequencies of alleles with $C H D$.

Material and methods: the Authors searched the literature on the peculiarities of IL-6 gene polymorphism in patients of different ethnic groups with ischemic heart disease, using the appropriate keywords in the search engines PubMed and Google Scholar, databases Scopus, Web of Science, MedLine, the Cochrane Library, EMBASE, Global Health, CyberLeninka, RSCI and others.

Results: the Potential relationship between IL-6 and IHD gene polymorphisms has been widely studied to date, but the results remain inconsistent and somewhat contradictory. The strongest relationship was established by genotypes IL-6-174G/C; IL-6-572G/C for different ethnic groups, which leads to the interest of studying in this direction in the era of individualization of medicine.

Practical implications: health care Organization, cardiology, internal diseases, pathological physiology.

Keywords: IL-6; gene polymorphism; cytokine regulation; coronary heart disease; atherosclerosis.

Сердечно-сосудистые заболевания (СС3) являются лидирующей причиной смертности во всем мире. По данным ВОЗ в 2000 г. ССЗ стали основной причиной смертности. В 1996 г. в мире от ССЗ умерло 15 млн. человек, по прогнозам экспертов к 2020 г. эта цифра может достичь 25 млн. Среди СС3 основной ущерб здоровью населения России и мира наносят ИБС и цереброваскулярная болезнь, поэтому усилия по профилактике и лечению в первую очередь должны быть направлены именно на эти две группы болезней, среди причин развития которых ведущее место занимает атеросклероз (АТ). 
Существует сильная ассоциация между системным воспалением и ИБС; эта ассоциация считается причинной, т. е. воспаление увеличивает риск заболевания, а не просто обозначает присутствие атеросклероза, который сам по себе является воспалительным процессом [1].

Основоположником клеточно-воспалительной гипотезы атеросклероза считается Рудольф Вирхов (R. Virchow), который в 1892 г. предложил термин «endarteriitis deformans nodosa», подчеркнув этим воспалительную природу заболевания, вовлечение в процесс внутренней оболочки сосудистой стенки с деформацией последней и очаговый (узловой) характер процесса; но представление об атеросклерозе, как о хроническом вялотекущем воспалительном заболевании, которое связано с дислипидемией и хронической иммунной дисрегуляцией, окончательно сформировалось лишь в XX столетии [2].

Согласно условиям доказательной медицины, маркеры воспаления в качестве предикторов риска развития сердечно-сосудистых заболеваний (СС3) и их осложнений, в настоящее время не соответствуют наивысшему уровню доказательности. Особого внимания заслуживает интерлейкин-6 (ИЛ-6), это мультифункциональный, плейотропный провоспалительный цитокин с молекулярной массой 26 кДа, синтезирующийся различными типами лимфоидных и нелимфоидных клеток, включая Т- и В-лимфоциты, фибробласты, эндотелиальные клетки, макрофаги/моноциты, мезангиальные и глиальные клетки, клетки синовиальной оболочки сустава, клетки жировой и опухолевой ткани [3]. Среди большого количества цитокинов, синтезируемых активированными Т-клетками, макрофагами и В-клетками, ключевая роль в развитии сердечно-сосудистых катастроф принадлежит именно ИЛ- 6 . Уровни ИЛ-6 являются прогностическими в отношении СС $3[2,4,5,6,7,8$, $9,10,11]$ и повышены у пациентов с нестабильной стенокардией по сравнению со стабильная стенокардия [12]. Сердечно-сосудистые факторы риска, такие как курение и физические упражнения, стресс приводят к повышению концентрации ИЛ-6 $[13,14,15,16,17,18]$.

Ген ИЛ-6 кодирует противовоспалительный цитокин в связи с этим повышение его концентрации в крови приводит к повышенной восприимчивости к развитию атеросклероза. Генотипы, в частности ИЛ-6-174G/G полиморфизма G-174С в промоторе гена ИЛ-6 (rs 1800795) повышают генную транскрипцию и в значимой степени ассоциированы с повышенными уровнями общего холестерина и ЛПНП [19], что и обуславливает влияние полиморфизма гена ИЛ-6 на метаболические факторы атерогенеза. Большое количество исследований показали, что генотип IL6-174G/C ассоци- 
ирован с высоким риском значительного стеноза коронарной артерии и является тем самым сильным предиктором ИБС.

В таблице 1 приведены 16 исследований по изучению генотипа IL-6174 G/C в разных странах у разных этнических групп, которая демонстрирует неоднородность в полученных данных, в большинстве исследований взаимосвязь с ИБС не установлена у жителей Южной Азии; положительна у жителей Америки и некоторых областей Европы.

Таблица 1.

Взаимосвязь генотипа IL-6-174 G/C с ИБС в разных странах с учетом этнических особенностей

\begin{tabular}{|c|l|c|l|l|}
\hline $\begin{array}{c}\text { № } \\
\text { п/п }\end{array}$ & \multicolumn{1}{|c|}{$\begin{array}{c}\text { Страна } \\
\text { исследования }\end{array}$} & $\begin{array}{c}\text { Год } \\
\text { публикации }\end{array}$ & $\begin{array}{l}\text { ИЛ-6 (генотип, } \\
\text { полиморфизм) }\end{array}$ & \multicolumn{1}{|c|}{$\begin{array}{c}\text { Связь с ИБС } \\
\text { и этническая связь }\end{array}$} \\
\hline 1 & Франция [20] & 2001 & IL-6-174 G/C & установлена \\
\hline 2 & Италия [21] & 2005 & IL-6-174 G>C & $\begin{array}{l}\text { установлена для аллеля } \\
\text { С у пожилых итальянцев }\end{array}$ \\
\hline 3 & Бразилия [22] & 2010 & IL-6-174 G>C & $\begin{array}{l}\text { у африканских бразиль- } \\
\text { цев установлена, у кав- } \\
\text { казских бразильцев нет }\end{array}$ \\
\hline 4 & Китай [23] & 2012 & IL-6-174 G/C & $\begin{array}{l}\text { у белых популяций } \\
\text { установлена, у восточ- } \\
\text { ных азиатов нет }\end{array}$ \\
\hline 5 & $\begin{array}{l}\text { Северная Aф- } \\
\text { рика [24] }\end{array}$ & 2013 & IL-6-174 G/C & установлена \\
\hline 6 & $\begin{array}{l}\text { Великобрита- } \\
\text { ния [25] }\end{array}$ & 2001 & IL-6-174 G/C & сомнительна \\
\hline 7 & США [26] & 2002 & IL-6-174 G/C & сомнительна \\
\hline 8 & США [27] & 2005 & IL-6-174 G>C & сомнительна \\
\hline 9 & Германия [28] & 2002 & IL-6-174 G/C & не установлена \\
\hline 10 & $\begin{array}{l}\text { Великобрита- } \\
\text { ния [29] }\end{array}$ & 2004 & IL-6-174 G>C & $\begin{array}{l}\text { не установлена для жи- } \\
\text { телей Северной Европы }\end{array}$ \\
\hline 11 & $\begin{array}{l}\text { Нидерланцы } \\
\text { [30] }\end{array}$ & 2006 & IL-6-174 G/C & не установлена \\
\hline 12 & Турция [31] & 2007 & IL-6-174 G/C & не установлена \\
\hline 13 & Германия [32] & 2004 & IL-6-174 G/C & $\begin{array}{l}\text { не установлена в кав- } \\
\text { казских образцах }\end{array}$ \\
\hline 14 & Тунис [33] & 2010 & IL-6-174 G>C & не установлена \\
\hline 15 & Турция [34] & 2011 & IL-6-174 G/C & не установлена \\
\hline 16 & Китай [35] & 2016 & ИЛ-6-174 G>C & не установлена \\
\hline
\end{tabular}


В таблице 2 приведены 11 исследований по изучению генотипа IL6-572G/C в разных странах у разных этнических групп, которая также демонстрирует неоднородность в полученных данных, в большинстве исследований взаимосвязь с ИБС не установлена у жителей Европы; в противоположность IL-6-174 G/C положительна у жителей Азиатских странах.

Таблица 2.

Взаимосвязь генотипа IL-6-572G/C с ИБС в разных странах с учетом этнических особенностей

\begin{tabular}{|c|l|c|l|l|}
\hline $\begin{array}{c}\text { № } \\
\text { п/I }\end{array}$ & \multicolumn{1}{|c|}{$\begin{array}{c}\text { Страна } \\
\text { исследования }\end{array}$} & $\begin{array}{c}\text { Год } \\
\text { публи- } \\
\text { кации }\end{array}$ & $\begin{array}{c}\text { ИЛ-6 (генотип, } \\
\text { полиморфизм) }\end{array}$ & $\begin{array}{c}\text { Связь с ИБС } \\
\text { и этническая связь }\end{array}$ \\
\hline 1 & Китай [36] & 2006 & IL-6-572 C/G & установлена \\
\hline 2 & Китай [37] & 2010 & IL-6-572 G/C & $\begin{array}{l}\text { установлена у китайцев } \\
\text { хань }\end{array}$ \\
\hline 3 & Мексика [38] & 2010 & $\begin{array}{l}\text { IL-6 -572 G>C } \\
\text { (rs1800796) }\end{array}$ & $\begin{array}{l}\text { установлена у мекси- } \\
\text { канских индивидов }\end{array}$ \\
\hline 4 & Китай [39] & 2011 & IL-6-572 C/G & установлена \\
\hline 5 & Турция [40] & 2015 & IL-6-572 G/C & $\begin{array}{l}\text { установлена } \\
\text { у родственников }\end{array}$ \\
\hline 6 & Китай [41] & 2006 & IL-6-572 C/G & $\begin{array}{l}\text { сомнительна у китай- } \\
\text { ского населения Ганса }\end{array}$ \\
\hline 7 & $\begin{array}{l}\text { Великобритания } \\
\text { [42] }\end{array}$ & 2001 & IL-6-572 G/C & не установлена \\
\hline 8 & $\begin{array}{l}\text { Великобритания } \\
\text { [29] }\end{array}$ & 2004 & IL-6-572 G>C & $\begin{array}{l}\text { не установлена у жите- } \\
\text { лей Северной Европы }\end{array}$ \\
\hline 9 & Швеция [43] & 2007 & IL-6-572 G>C & не установлена \\
\hline 10 & Индия [44] & 2009 & IL-6-572 G/C & $\begin{array}{l}\text { не установлена } \\
\text { уевероиндейского } \\
\text { населения }\end{array}$ \\
\hline 11 & Турция [34] & 2011 & IL-6-572 G/C & $\begin{array}{l}\text { не установлено } \\
\text { у турецкого населения }\end{array}$ \\
\hline
\end{tabular}

Таким образом проспективные клинические и эпидемиологические исследования показали, что повышение провоспалительного цитокина (ИЛ-6) свидетельствуют о более высоком риске и неблагоприятном прогнозе СС3, в том числе ИБС. Потенциальная связь между полиморфизмами генов ИЛ-6 и ИБС широко изучены на сегодняшний день, однако 
результаты исследований остаются непоследовательными и несколько противоречивыми, в связи с этим их изучение является крайне актуальным, ведь установление причинно-следственной связи между биомаркером и развитием ИБС и знание аллельных вариантов генов, которые могут влиять на уровень его экспрессии или особенности функционирования у различных этнических групп, позволит осуществлять профилактические меры в отношении пациентов группы риска.

\section{Сиисок литературы / References}

1. Ross R. Atherosclerosis - an inflammatory disease. N Engl J Med, 1998, Vol. 340, pp. 115-126.

2. Shal'novas A., Evstifeevas. E., Deev A.D., Metel'skaya V.A., Kapustina A.V i drugie. Vklad markerov vospaleniya i IBS v obshchuyu i serdechno-sosudistuyu smertnost' u pozhilyh zhitelej megapolisa (dannye issledovaniya SAHR) [Contribution of inflammation and ischemic heart disease markers to overall and cardiovascular mortality in the elderly population of the megalopolis (data of the SAHR study)]. Russ J Cardiol, 2015, №6 (122), pp. 7-13. DOI: http:// dx.doi.org/10.15829/1560-4071-2015-06-7-13.

3. Kishimoto T. The biology of interleukin-6. Blood, 1989, Vol. 71, pp. 1-10.

4. Harris T.B., Ferrucci L., Tracy R.P. et al. Mortality risk associated with elevated interleukin-6 and C-reactive protein in old age. Am J Med, 1999, Vol. 106, pp. 506-512.

5. Kabłak-Ziembicka A., Przewłocki T., Stępień E., Pieniążek P. et al. Relationship between carotid intima-media thickness, cytokines, atherosclerosis extent and a two-year cardiovascular risk in patients with arteriosclerosis. Kardiol Pol, 2011, Vol. 69(10), pp. 1024-1031.

6. Tanindi A., Sahinarslan A., Elbeg S., Cemri M. Relationship Between MMP1, MMP-9, TIMP-1, IL-6 and Risk Factors, Clinical Presentation, Extent and Severity of Atherosclerotic Coronary Artery Disease. Open Cardiovasc Med J, 2011, Vol. 5, pp. 110-116. doi: 10.2174/1874192401105010110.

7. Maitra A., Shanker J., Dash D., John S., Sannappa P.R., Rao V.S., et al. Polymorphisms in the IL6 gene in Asian Indian families with premature coronary artery disease--the Indian Atherosclerosis Research Study. Thromb Haemost, 2008, Vol. 99, pp. 944-950.

8. Brull D.J., Montgomery H.E., Sanders J., Dhamrait S., Luong L., Rumley A., et al. Interleukin- 6 gene- $174 \mathrm{G} / \mathrm{C}$ and $-572 \mathrm{G} / \mathrm{C}$ promoter polymorphisms are strong predictors of plasma interleukin-6 levels after coronary artery bypass surgery. Arterioscler Thromb Vasc Biol, 2001, Vol. 21, pp.1458-1463. 
9. Isaac Subirana, Montserrat Fitó, Oscar Diaz, Joan Vila, Albert Francés, Eva Delpon, Juan Sanchis, Roberto Elosua, Daniel Muñoz-Aguayo, Irene R. Dégano\&Jaume Marrugat. Prediction of coronary disease incidence by biomarkers of infammation, oxidation, and metabolism. Scientific Reports, 2018, Vol. 8, pp.3191. DOI:10.1038/s41598-018-21482-y.

10. Bernhard Schieffer; Tina Selle; Andres Hilfiker; Denise Hilfiker-Kleiner, et all. Impact of Interleukin-6 on Plaque Development and Morphology in Experimental Atherosclerosis. Circulation, 2004, Vol. 110, pp. 3493-3500. DOI:10.1161/01. CIR.0000148135.08582.97.

11. Mitrokhin V., Nikitin A., Brovkina O. et all. Association between interleukin-6/6R gene polymorphisms and coronary artery disease in Russian population: influence of interleukin-6/6R gene polymorphisms on inflammatory markers. Journal of Inflammation Research, 2017, Vol. 10, pp. 151-160.

12. Biasucci L.M., Vitelli A., Liuzzo G. Elevated levels of IL-6 in unstable angina. Circulation, 1996, Vol. 94, pp. 874-877.

13. de Maat M.P., Pietersma A., Kofflard M., Sluiter W., Kluft C. Association of plasma fibrinogen levels with coronary artery disease, smoking and inflammatory markers. Atherosclerosis, 1996, Vol. 121, pp. 185-191.

14. Tappia P.S., Troughton K.L., Langley-Evans S.C., Grimble R.F. Cigarette smoking influences cytokine production and anti-oxidant defenses. Clin Sci, 1995, Vol. 88, pp. 485-489.

15. Northoff H., Weinstock C., Berg A. The cytokine response to strenuous exercise. Int J Sports Med, 1994, Vol. 15, pp. 167-171.

16. Zhou D., Kusnecov A.W., Shurin M.R., DePaoli M., Rabin B.S. Exposure to physical and psychological stressors elevates plasma interleukin-6: relationship to the activation of the hypothalamic-pituitary-adrenal axis. Endocrinology, 1993, Vol. 133, pp. 2523-2530.

17. Maes M., Song C., Lin A. et al. The effects of psychological stress on humans: increased production of pro-inflammatory cytokines and a Th1-like response in stress-induced anxiety. Cytokine, 1998, Vol.10, pp. 313-318.

18. Song C., Kenis G., van Gastel A. et al. Influence of psychological stress on immune-inflammatory variables in normal humans. Part II. Altered serum concentrations of natural anti-inflammatory agents and soluble membrane antigens of monocytes and T lymphocytes. Psychiatry Res, 1999, Vol.85, pp. 293-303.

19. Riikola, A. et al. Interleukin-6 promoter polymorphism and cardiovascular risk factors: the Health 2000 Survey. Atherosclerosis, 2009, Vol. 207, №2, pp. 466-470.

20. Georges J.L., Loukaci V., Poirier O., Evans A., Luc G., Arveiler D. et al. Interleukin- 6 gene polymorphisms and susceptibility to myocardial infarction: the 
ECTIM study. Etude Cas-Témoin de l'Infarctus du Myocarde. J Mol Med Berl, 2001, Vol. 79(5-6), pp. 300-305.

21. Chiappelli M., Tampieri C., Tumini E., Porcellini E., Caldarera C.M., Nanni S. et al. Interleukin-6 gene polymorphism is an age-dependent risk factor for myocardial infarction in men. Int J Immunogenet, 2005, Vol. 32(6), pp. 349-353.

22. Rios D.L., Cerqueira C.C., Bonfim-Silva R., Araújo L.J., Pereira J.F., Gadelha S.R. et al. Interleukin-1 beta and interleukin-6 gene polymorphism associations with angiographically assessed coronary artery disease in Brazilians. Cytokine, 2010, Vol. 50(3), pp. 292-296.

23. Niu W., Liu Y., Qi Y., Wu Z., Zhu D., Jin W. Association of interleukin-6 circulating levels with coronary artery disease: a meta-analysis implementing Mendelian randomization approach. Int J Cardiol, 2012, Vol. 157(2), pp. 243-252.

24. Phulukdaree A., Khan S., Ramkaran P., Govender R., Moodley D., Chuturgoon A.A. The interleukin- $6-147 \mathrm{~g} / \mathrm{c}$ polymorphism is associated with increased risk of coronary artery disease in young South African Indian men. Metab Syndr Relat Disord, 2013, Vol. 11, pp. 205-209.

25. Humphries S.E., Luong L.A., Ogg M.S., Hawe E., Miller G.J. The interleukin-6-174 G/C promoter polymorphism is associated with risk of coronary heart disease and systolic blood pressure in healthy men. Eur Heart J, 2001, Vol. 22(24), pp. 2243-2252.

26. Jenny N.S., Tracy R.P., Ogg M.S., le Luong A., Kuller L.H., Arnold A.M. et al. In the elderly, interleukin- 6 plasma levels and the $-174 \mathrm{G}>\mathrm{C}$ polymorphism are associated with the development of cardiovascular disease. Arterioscler Thromb Vasc Biol, 2002, Vol, 22(12), pp. 2066-2071.

27. Rosner S.A., Ridker P.M., Zee R.Y., Cook N.R. Interaction between inflammation-related gene polymorphisms and cigarette smoking on the risk of myocardial infarction in the physician's health study. Hum Genet, 2005, Vol. 118(2), pp. 287-294.

28. Nauck M., Winkelmann B.R., Hoffmann M.M., Böhm B.O., Wieland H., März W. The interleukin-6 G(-174)C promoter polymorphism in the LURIC cohort: no association with plasma interleukin-6, coronary artery disease, and myocardial infarction. J Mol Med Berl, 2002, Vol. 80(8), pp. 507-513.

29. Kelberman D., Hawe E., Luong L.A., Mohamed-Ali V., Lundman P., Tornvall P. et al. Effect of Interleukin-6 promoter polymorphisms in survivors of myocardial infarction and matched controls in the North and South of Europe. The HIFMECH Study. Thromb Haemost, 2004, Vol. 92(5), pp. 1122-1128.

30. Sie M.P., Sayed-Tabatabaei F.A., Oei H.H., Uitterlinden A.G., Pols H.A., Hofman A. et al. Interleukin 6-174 g/c promoter polymorphism and risk of 
coronary heart disease: results from the rotterdam study and a meta-analysis. Arterioscler Thromb Vasc Biol, 2006, Vol. 26(1), pp. 212-217.

31. Sekuri C., Cam F.S., Sagcan A., Ercan E., Tengiz I., Alioglu E., et al. No association of interleukin- 6 gene polymorphism $(-174 \mathrm{G} / \mathrm{C})$ with premature coronary artery disease in a Turkish cohort. Coron Artery Dis, 2007, Vol. 18, pp. 333-337.

32. Lieb W., Pavlik R., Erdmann J., Mayer B., Holmer S.R., Fischer M. et al. No association of interleukin- 6 gene polymorphism $(-174 \mathrm{G} / \mathrm{C})$ with myocardial infarction or traditional cardiovascular risk factors. Int J Cardiol, 2004, Vol. 97(2), pp. 205-212.

33. Ghazouani L., Ben Hadj Khalifa S., Abboud N., Ben Hamda K., Ben Khalfallah A., Brahim N. et al. TNF-alpha $-308 \mathrm{G}>\mathrm{A}$ and IL-6 -174G $>$ C polymorphisms in Tunisian patients with coronary artery disease. Clin Biochem, 2010, Vol. 43(13-14), pp. 1085-1089.

34. Coker A., Arman A., Soylu O., Tezel T., Yildirim A. Lack of association between IL-1 and IL-6 gene polymorphisms and myocardial infarction in Turkish population. Int J Immunogenet, 2011, Vol. 38(3), pp. 201-208.

35. Hongmei Y., Yongping J., Jiyuan L. Interleukin-6 polymorphisms and risk of coronary artery diseases in a Chinese population: A casecontrol study. Pak J Med Sci, 2016, Vol. 32(4), pp. 880-885. doi: http://dx.doi.org/10.12669/ pjms.324.9908.

36. Wei Y.S., Lan Y., Liu Y.G., Tang R.G., Lan J.S. Relationship between interleukin-6 gene polymorphism and coronary heart disease and its effect on plasma lipid levels. Zhongguo Wei Zhong Bing Ji Jiu Yi Xue, 2006, Vol. 18(4), pp. 233-236.

37. Jia X., Tian Y., Wang Y., Deng X., Dong Z., Scafa N. et al. Association between the interleukin- 6 gene $-572 \mathrm{G} / \mathrm{C}$ and $-597 \mathrm{G} / \mathrm{A}$ polymorphisms and coronary heart disease in the Han Chinese. Med Sci Monit, 2010, Vol. 16(3), pp.103-108.

38. Fragoso J.M., Delgadillo H., Juárez-Cedillo T., Rodríguez-Pérez J.M., Vallejo M., Pérez-Méndez O. et al. The interleukin 6-572 G > C (rs1800796) polymorphism is associated with the risk of developing acute coronary syndrome. Genet Test Mol Biomarkers, 2010, Vol. 14(6), pp. 759-763.

39. Fan W.H., Liu D.L., Xiao L.M., Xie C.J., Sun S.Y., Zhang J.C. Coronary heart disease and chronic periodontitis: is polymorphism of interleukin-6 gene the common risk factor in a Chinese population? Oral Dis, 2011, Vol. 17(3), pp. 270-276.

40. Ataç Çelik, Mustafa Özçetin, Ömer Ateş, Fatih Altunkaş, et al. Analyses of C-Reactive Protein, Endothelial Nitric Oxide Synthase and Interleukin-6 Gene Polymorphisms in Adolescents with a Family History of Premature Coronary Artery Disease: A Pilot Study. Balkan Medical Journal, 2015, Vol 32(4), pp. 397-402. 
41. Fu H.X., Li G.S., Li Y., Xu J.L., Zhang J.Y. Interleukin-6 -597G/A and -572C/ G polymorphisms and risk of coronary heart disease. Zhonghua Xin Xue Guan Bing Za Zhi, 2006, Vol. 34(6), pp. 519-522.

42. Humphries S.E., Luong L.A., Ogg M.S., Hawe E., Miller G.J. The interleukin-6 $-174 \mathrm{G} / \mathrm{C}$ promoter polymorphism is associated with risk of coronary heart disease and systolic blood pressure in healthy men. Eur Heart J, 2001, Vol. 22(24), pp. 2243-2252.

43. Mälarstig A., Wallentin L., Siegbahn A. Genetic variation in the interleukin-6 gene in relation to risk and outcomes in acute coronary syndrome. Thromb Res, 2007, Vol. 119(4), pp. 467-473.

44. Banerjee I., Pandey U., Hasan O.M., Parihar R., Tripathi V., Ganesh S. Association between inflammatory gene polymorphisms and coronary artery disease in an Indian population. $J$ Thromb Thrombolysis, 2009, Vol. 27(1), pp. 88-94.

\section{ДАННЫЕ ОБ АВТОРАХ}

Смирнова Ольга Валентиновна, д.м.н., профессор, заведующая лабораторией клинической патофизиологии Федеральное государственное бюджетное научное учреждение «Федеральный исследовательский центр «Красноярский научный иентр Сибирского отделения Российской академии наук» (КНЦ СО РАН, ФИЦ КНЦ СО РАН) «Научно-исследовательский институт медицинских проблем Севера»

ул. Партизана Железняка, 32, г. Красноярск, 660022, Российская Федераиия ovsmirnova71@mail.ru

Каблуков Виталий Сергеевич, аспирант 2 года обучения по направлению 30.06.01 - Фундаментальная медицина по научной специальности 14.03 .03 - Патологическая физиология

Федеральное государственное бюджетное научное учреждение "Федеральный исследовательский иентр «Красноярский научный иентр Сибирского отделения Российской академии наук» (КНЦ СО РАН, ФИЦ КНЦ СО РАН) «Научно-исследовательский институт медичинских проблем Севера»

ул. Партизана Железняка, 32, г. Красноярск, 660022, Российская Федераиия

vitala600@yandex.ru 
ORCID: 0000-0002-2765-6726

SPIN-код: 3261-5064

\section{DATA ABOUT THE AUTHORS}

Smirnova Olga Valentinovna, MD, Professor, Head of the Laboratory of Clinical Pathophysiology

Federal Research Center «Krasnoyarsk Science Center SB RAS» «Scientific Research Institute of Medical Problems of the North»

3d, Partizan Zheleznyaka Str., Krasnoyarsk, 660022, Russian Federation ovsmirnova71@mail.ru

Kablukov Vitaly Sergeevich, Postgraduate of 2 year of study in the direction 30.06.01-Fundamental medicine in the scientific specialty 14.03.03-Pathological physiology

Federal Research Center «Krasnoyarsk Science Center SB RAS» «Scientific Research Institute of Medical Problems of the North»

3d, Partizan Zheleznyaka Str., Krasnoyarsk, 660022, Russian Federation vitala600@yandex.ru

ORCID: 0000-0002-2765-6726

SPIN-cod: 3261-5064 